\title{
Efficient electron injection into plasma waves using higher-order laser modes
}

\author{
P. Michel, E. Esarey, ${ }^{*}$ C. B. Schroeder, B. A. Shadwick, and W. P. Leemans* \\ Lawrence Berkeley National Laboratory, Berkeley, California 94720, USA
}

(Dated: August 2, 2006)

\begin{abstract}
Using higher-order transverse laser modes as drivers for plasma wave excitation, and, in particular, using a ring laser beam with maximum intensity off-axis, results in reversal of the focusing and defocusing phase regions in a laser wakefield accelerator. This results in improved performance of self-trapping and laser injection schemes. Specifically, the trapping threshold required for optical injection is decreased and the maximum energy gain of the trapped electrons is increased. This scheme could also be of interest for the generation of ring electron beams or for beam conditioning.
\end{abstract}

\footnotetext{
*Also at University of Nevada, Reno
} 


\section{INTRODUCTION}

Laser-plasma-based accelerators [1] have generated great interest recently, due to a series of experimental breakthroughs leading to the production of quasi-monoenergetic electron beams with energies $\gtrsim 100 \mathrm{MeV}[2-4]$. These experiments relied on self-trapping of background plasma electrons, which requires sufficiently large plasma waves and, hence, sufficiently large drive laser intensities (typically, $I \geq 10^{19} \mathrm{~W} / \mathrm{cm}^{2}$, where $I$ is the peak laser intensity). However, the self-trapping mechanism is difficult to control, since it relies on highly nonlinear plasma processes. Therefore, several methods have been proposed [5-13] in order to provide controlled injection of background plasma electrons. Optical injection schemes $[5,6,8,9,11-13]$ are particularly promising. For example, colliding pulse injection (CPI) $[6,8,9]$, as originally proposed, used three laser pulses: two counterpropagating pulses in addition to the drive pulse that generates the wakefield. The two auxiliary pulses will collide at some predetermined phase in the wakefield of the drive pulse, and the slow phase velocity beat wave generated during the collision of the two auxiliary pulses can inject background plasma electrons into the wakefield. Colliding pulse injection can be achieved using only two laser pulses $[11,12]$, in which the second, counterpropagating laser pulse collides with the pump pulse to inject electrons into the wakefield near the back of the pump pulse.

One universal difficulty with all previous injection schemes (self-trapped and external) is that the electrons are initially injected on orbits that are not only accelerating but defocusing. In one-dimension (1D), an electron becomes trapped when it is injected onto a phase space orbit lying above the separatrix. The separatrix is the orbit that separates the region of untrapped (or open) orbits from the region of trapped (or closed) orbits in phase space. When two-dimensional (2D) effects are taken into account, the trapped orbits lying closest to the 1D separatrix are also defocusing, i.e., the electrons quickly phase slip from a region that is both accelerating and focusing to a region that is both accelerating but defocusing (for a linear plasma wave, the region of overlap between the accelerating and focusing fields is one-quarter of a plasma period). To ensure that electrons remain on orbits that are entirely accelerating and focusing (up to the limits of dephasing) requires that the electrons be injected on "deeply" trapped orbits lying sufficiently above the 1D separatrix. This means that the electrons must be injected with high energy, which requires high laser intensities.

In this paper, the use of higher-order transverse laser modes for plasma wave excitation 
is examined. In particular, the use of a combination of modes is considered such that the transverse laser intensity gradient is positive near the propagation axis (i.e., $\partial I / \partial r>0$ ). This can result in a complete inversion of the the focusing and defocusing phase regions. For example, linear theory predicts that the wakefield excited by a laser pulse is described by an electrostatic potential of the form $\phi(r, z) \sim \hat{\phi}(r) \sin k_{p} \zeta$, where $\zeta=z-v_{g} t, v_{g}$ is the wake phase velocity (approximately equal to the group velocity $v_{g}$ of the drive laser pulse), $\hat{\phi}(r)$ is proportional to the transverse laser intensity profile, $k_{p}=2 \pi / \lambda_{p}=\omega_{p} / c$, and $\omega_{p}$ is the electron plasma frequency. If the intensity profile is peaked on axis $(\partial \hat{\phi} / \partial r<0)$, then, e.g., the phase region $-9 \pi / 2<k_{p} \zeta<-7 \pi / 2$ is accelerating $\left(E_{z}=-\partial \phi / \partial z<0\right)$, as shown in Fig. 1, but only the region $-4 \pi<k_{p} \zeta<-7 \pi / 2$ is accelerating and focusing $\left(E_{r}=-\partial \phi / \partial r<0\right)$. Electrons that are initially injected into the focusing and accelerating region $\left(-4 \pi<k_{p} \zeta<-7 \pi / 2\right)$ at low energy will quickly phase slip (backward with respect to the high phase velocity wakefield) into the defocusing region $\left(-9 \pi / 2<k_{p} \zeta<-4 \pi\right)$ before acceleration to high energies has occurred. On the other hand, if the intensity profile has a minimum on axis $\left(\partial \hat{\phi} / \partial r>0\right.$ near the axis), then the phase region $-9 \pi / 2<k_{p} \zeta<-4 \pi$ is both accelerating and focusing. As will be shown in the following sections, this implies that electrons can be injected on trapped and focused orbits closer to the 1D separatrix, thus allowing injection and trapping with lower electron energies and lower laser intensities. In addition, it is shown that if the trapped electron bunch is allowed to be accelerated over sufficiently long distances, then the trapped bunch will naturally evolve into a transverse profile with a ring shape.

The remainder of this paper is organized as follows. In Sec. II we describe how the transverse laser profile affects the electron phase space dynamics. In Sec. III we present numerical examples of the electron dynamics, showing the improved efficiency of optical injection with a higher-order mode drive laser. Discussion of these results and applications of wakefields excited by higher-order laser modes is presented in Sec. IV.

\section{PHASE SPACE ANALYSIS}

In the linear cold plasma regime [14], the longitudinal component of the laser-driven plasma wakefield is a simple sinusoidal function, while its transverse profile is proportional to the drive laser pulse intensity profile. The excited wakefield can be approximated by the 
linear regime result provided $\left\langle a^{2}\right\rangle<1$, where $a \simeq 8.6 \times 10^{-10} \lambda_{0}[\mu \mathrm{m}] I^{1 / 2}\left[\mathrm{~W} / \mathrm{cm}^{2}\right]$ is the laser strength parameter for a linear polarized laser with $I$ the laser intensity and $\lambda_{0}$ the laser wavelength (the angular brackets denote the time-average). The cold plasma fluid approximation will be valid provided the electrostatic field of the plasma wakefield is below the wavebreaking limit $[15,16]$. For a laser pulse with a Gaussian transverse profile, the excited plasma wave potential in cylindrical coordinates $(r, z)$ is $\phi(r, \zeta)=\phi_{0} \exp \left(-2 r^{2} / r_{0}^{2}\right) \sin \left(k_{p} \zeta\right)$, where $\phi_{0}$ is the wakefield amplitude, $r_{0}$ is the laser pulse transverse spot size, $\zeta=z-v_{g} t$, and $v_{g} \simeq c\left(1-\omega_{p}^{2} / 2 \omega_{0}^{2}-2 c^{2} / \omega_{0}^{2} r_{0}^{2}\right)$ is the laser group velocity, with $\omega_{0}$ is the laser frequency, assuming $\omega_{p} / \omega_{0} \ll 1$ and $\omega_{0} r_{0} / c \gg 1$. The potential is normalized so that $\phi=e \Phi / m c^{2}$ (where $m$ is the electron mass and $e$ is the electron charge), where $\Phi$ is the electrostatic potential.

The motion of an electron in the linear electrostatic plasma wakefield is given by $d \boldsymbol{u} / d c t=$ $\boldsymbol{\nabla} \phi$, where $\boldsymbol{u}=\boldsymbol{p} / m c$ is the normalized momentum. The longitudinal electron orbit phase space $\left(\zeta, u_{z}\right)$ is shown in Fig. 1. The $1 \mathrm{D}$ separatrix defines the limit between the trapped and untrapped orbits in the wakefield, assuming 1D motion of the particles in the longitudinal electric field associated with the wakefield, $E_{z}=-\partial \Phi / \partial z$. However, when 2D effects (i.e., the non-uniform transverse pulse profile) are included, there is a radial electric field $E_{r}=$ $-\partial \Phi / \partial r$ that is alternatively focusing and defocusing in a $\lambda_{p} / 2$ phase region. The transverse and longitudinal fields are dephased by $\pi / 2$, so that there exists a $\lambda_{p} / 4$ phase region that is both focusing and accelerating for the electrons. For a drive laser pulse with a Gaussian transverse profile, electrons trapped above the 1D separatrix (and below the 2D separatrix) will slip into a defocusing phase region and will be expelled radially. Electrons will be trapped and focused on orbits within the $2 \mathrm{D}$ separatrix of longitudinal extent $\lambda_{p} / 2$ (cf. Fig. 1).

As it can be seen in Fig. 1, the momentum required for the particles to move from the cold fluid orbit (which corresponds to the orbits of electrons initially at rest before the passage of the drive laser) to the $2 \mathrm{D}$ separatrix is much higher than that required to move above the 1D separatrix. By using higher-order transverse laser modes, and in particular laser pulses with a transverse "ring" profile with a peak amplitude off-axis, the longitudinal phase regions that were defocusing for a Gaussian transverse profile become focusing near the axis, and vice-versa. This will allow electrons to be trapped and focused above the 1D separatrix and, hence, dramatically lower the trapping threshold. Furthermore, it will be 


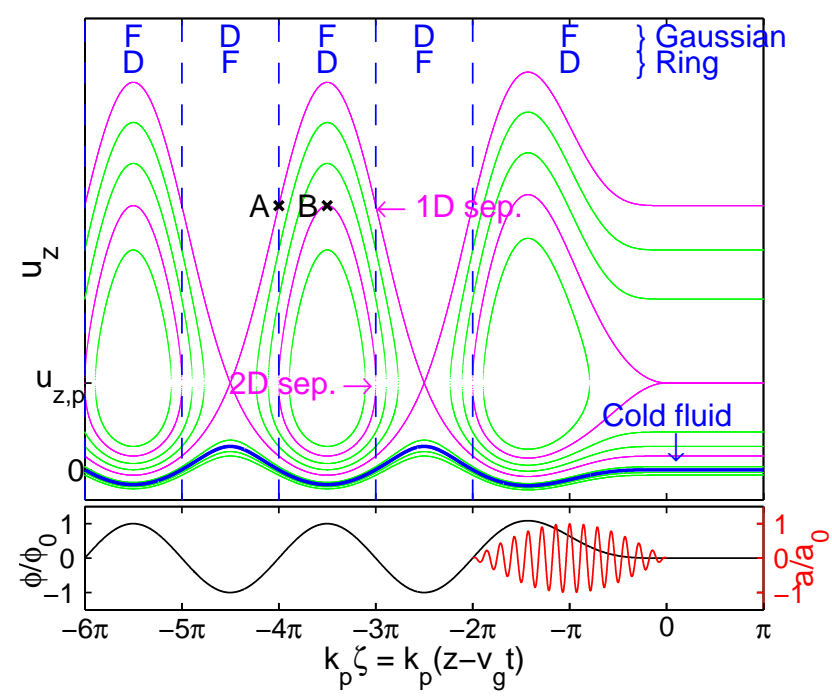

FIG. 1: Phase-space diagram showing the 1D and 2D separatrices and the cold fluid orbit. Also plotted at the bottom are the laser pulse and the plasma wakefield longitudinal profile. Defocusing (D) and focusing (F) phase regions are shown for Gaussian and ring laser profiles.

shown that once an electron on the 1D separatrix reaches the point A in Fig. 1 (at phase $\left.k_{p} \zeta=-4 \pi\right)$, it still remains focused into the off-axis ring of the excited wakefield, where the peak amplitude of the wakefield is maximum. This allows the electrons to reach higher energies, and provides a method to shape the transverse profile of the electron beam into a ring profile, which could have applications for efficient radiation generation as described in Sec. IV.

\section{A. Wakefield excitation using higher-order laser modes}

Laguerre-Gaussian (LG) modes constitute a complete set of eigenfunctions describing the solution to the paraxial wave equation [17]. Near the focal point, i.e., $|z| \ll Z_{R}=\pi r_{0}^{2} / \lambda_{0}$, where $Z_{R}$ is the Rayleigh length and $r_{0}$ is the focal spot size, axisymmetric LG modes are characterized by a transverse spatial profile of the form $a \propto L_{m}(s) \exp [-s / 2]$, where $L_{m}$ is the Laguerre polynomial of order $m$ and $s=2 r^{2} / r_{0}^{2}$. A linear combination of the zeroth and first axisymmetric modes $\left(\mathrm{LG}_{0}\right.$ and $\left.\mathrm{LG}_{1}\right)$ can lead to a transversely ring-shaped beam (with nonzero intensity on axis). This higher-order mode laser field has $\partial a^{2} / \partial r>0$ near the axis (opposite to that of a Gaussian mode with $\partial a^{2} / \partial r<0$ ). Throughout this study, the normalized transverse vector potential $\left(a=e A_{\perp} / m c^{2}\right)$ of the drive laser pulse is assumed to 
have the form

$$
a(r, z, t)=a_{0}\left(\alpha+\beta \frac{r^{2}}{r_{0}^{2}}\right) e^{-r^{2} / r_{0}^{2}} \sin \left(\pi \zeta / L_{0}\right) \cos \left(\psi_{0}\right),
$$

for $-L_{0} \leq \zeta \leq 0$, and 0 elsewhere (corresponding to a half-sine longitudinal laser profile of length $\left.L_{0}\right)$. Here $\psi_{0}=k_{0}\left(z-v_{p} t\right)$ and $v_{p}$ is the phase velocity of the laser. The parameters $\alpha$ and $\beta$ determine the weights of the zeroth and first LG modes, and define the transverse profile of the pulse. Equation (1) is valid near the focal point, $|z| \ll Z_{R}$. Neglected in Eq. (1) are the effects of diffraction and small differences in the group and phase velocities between the fundamental and first order LG modes. The effects of diffraction can be overcome by guiding the laser pulses within a broad $\left(k_{p} r_{\perp} \gg 1\right)$ plasma channel. Differences in the phase velocities of the two modes will cause the phases to slip by a laser period over a distance on the order of $Z_{R}$. Hence, the normalized peak laser intensity given by $a^{2} \propto$ $a_{0}^{2}\left(\alpha+\beta r^{2} / r_{0}^{2}\right)^{2}$ is only valid for $|z| \ll Z_{R}$, assuming the two modes are polarized in the same direction, as assumed in Eq. (1). Mode dephasing issues can be overcome by using orthogonal polarizations in the two modes. For orthogonal polarization, the peak laser intensity is $a^{2} \propto a_{0}^{2}\left(\alpha^{2}+\beta^{2} r^{4} / r_{0}^{4}\right)$, as opposed to $a^{2} \propto a_{0}^{2}\left(\alpha+\beta r^{2} / r_{0}^{2}\right)^{2}$ for parallel polarization (which is assumed in the following). The peak intensity is the relevant parameter, since it is the intensity profile that drives the plasma wave (accelerating and focusing forces) via the ponderomotive force.

The wakefield associated to this drive beam can be calculated using the quasistatic approximation for a linear wakefield [18]:

$$
\phi=k_{p} \int d \zeta^{\prime} \sin k_{p}\left(\zeta-\zeta^{\prime}\right) a^{2}\left(\zeta^{\prime}\right) / 2,
$$

where we have averaged over the fast laser oscillations. Using Eq. (2) with the laser field Eq. (1) yields the following expression for the wakefield:

$$
\phi(r, z, t)=\phi_{0}\left(\alpha+\beta \frac{r^{2}}{r_{0}^{2}}\right)^{2} e^{-2 r^{2} / r_{0}^{2}} \phi_{\zeta},
$$

with $\phi_{0}=\pi a_{0}^{2} / 8$. For the resonant case where the pulse length equals the plasma wavelength, $L_{0}=\lambda_{p}$,

$$
\phi_{\zeta}=\frac{1}{\pi}\left[1-\cos \left(k_{p} \zeta\right)-\frac{1}{2} k_{p} \zeta \sin \left(k_{p} \zeta\right)\right],
$$

inside the pulse $\left(-2 \pi \leq k_{p} \zeta \leq 0\right)$, and $\phi_{\zeta}=\sin \left(k_{p} \zeta\right)$ behind the pulse $\left(k_{p} \zeta \leq-2 \pi\right)$. 
It is useful to normalize the laser field with respect to the power contained in the laser beam $P=\int I(r) r d \theta d r$, where $I(r)=\left[m^{2} c^{3} \omega_{0}^{2} /\left(8 \pi e^{2}\right)\right] a(r)^{2}$ is the laser intensity. We will use the following notation: $I=I_{0} a_{r}^{2}$, where $I_{0}=\left[m^{2} c^{3} \omega_{0}^{2} /\left(8 \pi e^{2}\right)\right] a_{0}^{2}$ and $a_{r}=\left(\alpha+\beta r^{2} / r_{0}^{2}\right) \exp \left[-r^{2} / r_{0}^{2}\right]$. The laser power in the mode described by Eq. (1) is

$$
P=\frac{\pi r_{0}^{2} I_{0}}{2}\left(\alpha^{2}+\alpha \beta+\beta^{2} / 2\right) \text {. }
$$

A Gaussian profile corresponds to $\alpha=1$ and $\beta=0$; in this case we recover the usual expression $P=\pi r_{0}^{2} I_{0} / 2$. For the case of a hollow ring with $a(r=0)=0, \alpha=0$. Constant laser power for transverse laser profiles (defined by $\alpha$ and $\beta$ ) requires $\alpha$ and $\beta$ to satisfy $\alpha^{2}+\alpha \beta+\beta^{2} / 2=1$, or, for $\alpha \in[0,1], \beta=-\alpha+\sqrt{2-\alpha^{2}}$. Note that a flat-top transverse profile corresponds to $\alpha_{\text {flat }}=(2 / 5)^{1 / 2}$.

\section{B. Trapping thresholds}

The Hamiltonian for an electron in the plasma wakefield is given by $[8,19] H=\gamma-$ $\beta_{g} u_{z}-\phi$, where $\gamma=\left(1-v^{2} / c^{2}\right)^{-1 / 2}$ is the relativistic Lorentz factor of the electron, and $\beta_{g}=v_{g} / c$. From this Hamiltonian we can derive the longitudinal momentum, assuming no laser field is present,

$$
u_{z}=\beta_{g} \gamma_{g}^{2}(H+\phi) \pm \gamma_{g} \sqrt{\gamma_{g}^{2}(H+\phi)^{2}-1},
$$

where $\gamma_{g}=\left(1-\beta_{g}^{2}\right)^{-1 / 2}$ is the Lorentz factor associated to the group velocity of the laser (which is approximately the phase velocity of the wakefield).

For simplicity we have assumed 1D motion of the electrons, $u_{x}=u_{y}=0$. The Hamiltonian for the $1 \mathrm{D}$ and $2 \mathrm{D}$ separatrices, within the linear cold fluid approximation where the longitudinal component of the wakefield is a sine function of amplitude $\phi_{0}$, are given by $H_{s 1 \mathrm{D}}=1 / \gamma_{g}+\alpha^{2} \phi_{0}$ and $H_{s 2 \mathrm{D}}=1 / \gamma_{g}$ (where the factor $\alpha^{2}$ accounts for the fact that we assume propagation on axis). The cold fluid orbit (corresponding to an electron initially at rest) is defined by the Hamiltonian value $H=1$.

As shown in Fig. 1, using a higher-order mode laser driver, the focusing and defocusing phase regions can be exchanged, compared to the usual Gaussian laser case (or any similar profile peaked on axis). To achieve this exchange of focusing regions near the axis requires a wakefield excited by a laser profile that satisfies $0 \leq \alpha<(2 / 5)^{1 / 2}$. 
The threshold laser intensity required for trapping using the two-pulse or three-pulse CPI schemes can be calculated by using a Hamiltonian analysis. In the beatwave, when no wakefield is present $(\phi=0)$ the Hamiltonian describing the electron motion is $H_{b}=\gamma-\beta_{b} u_{z}$, where $\beta_{b}=\Delta \omega / c \Delta k$ is the beat wave velocity normalized to $c$. Here, $\Delta \omega=\omega_{i}-\omega_{j}$ and $\Delta k=k_{i}-k_{j}$ are the differences between the forward-going (subscript $i$ ) and backwardgoing (subscript $j$ ) lasers frequencies and wavenumbers, respectively. In the following, the subscript 0 will be used for the drive pulse, 1 for the counterpropagating injection pulse, and 2 for the forward-going injection pulse (present in three-pulse CPI only). The relativistic Lorentz factor of the electron in the beatwave is given by $\gamma^{2}=\gamma_{\perp}^{2}+u_{z}^{2}$, where $\gamma_{\perp}^{2}=$ $1+a_{i}^{2} / 2+a_{j}^{2} / 2+a_{i} a_{j} \cos \left(\psi_{b}\right), a_{i}$ and $a_{j}$ are the amplitudes of the colliding pulses, and $\psi_{b}=\Delta k\left(z-\beta_{b} c t\right)$ is the beatwave phase.

From the Hamiltonian $H_{b}$ the expression of the momentum of a particle on the beatwave separatrix (with Hamiltonian $H_{b s}=\gamma_{\perp}(0) / \gamma_{b}[8]$ ) can be derived. The extrema values of the longitudinal momentum $u_{b \pm}$ and relativistic Lorentz factor $\gamma_{b \pm}$ on the separatrix are

$$
\begin{aligned}
& u_{b \pm}=\gamma_{b} \beta_{b} \gamma_{\perp}(0) \pm \sqrt{2} \gamma_{b} \sqrt{a_{i} a_{j}} \\
& \gamma_{b \pm}=\gamma_{\perp}(0) \gamma_{b} \pm \sqrt{2} \gamma_{b} \beta_{b} \sqrt{a_{i} a_{j}}
\end{aligned}
$$

where $\gamma_{b}=\left(1-\beta_{b}\right)^{-1 / 2}$ is the relativistic Lorentz factor associated with the beatwave phase velocity.

For the three-pulse CPI, it is possible to derive the trapping threshold as the value of $\sqrt{a_{1} a_{2}}$ that allows for an electron on the cold fluid orbit $(H=1)$ to move above the wakefield separatrix onto a trapped orbit $\left(H<H_{s}\right.$, where $H_{s}$ is the Hamiltonian for the $1 \mathrm{D}$ or 2D separatrix). The expression for the threshold amplitude is [8]

$$
{\sqrt{a_{1} a_{2}}}_{\mathrm{th}}=\frac{\left(1-H_{s}\right)}{\sqrt{2} \gamma_{b}\left(\beta_{g}-\beta_{b}\right)},
$$

where $H_{s}$ can be evaluated for the $1 \mathrm{D}$ or $2 \mathrm{D}$ separatrix. The ratio of the thresholds for injection above the $1 \mathrm{D}$ separatrix and $2 \mathrm{D}$ separatrix is

$$
\frac{{\sqrt{a_{1} a_{2}}}_{\mathrm{th}, 1 \mathrm{D}}}{{\sqrt{a_{1} a_{2}}}_{\mathrm{th}, 2 \mathrm{D}}}=1-\frac{\phi_{0}}{1-1 / \gamma_{g}} .
$$

For an underdense plasma with $\gamma_{g} \gg 1$, the ratio is $1-\phi_{0}$. This shows that in order to lower the trapping threshold compared to the Gaussian drive laser case, one has to increase 
the drive beam intensity in order to increase $\phi_{0}$ to values close to unity (i.e., close to the non-linear regime). For example, if $\phi_{0}=0.9$, and assuming $\gamma_{g} \gg 1$, the threshold for $\sqrt{a_{1} a_{2}}$ is decreased by an order of magnitude compared to the Gaussian wakefield case.

The trapping threshold for the two-pulse CPI scheme is more difficult to evaluate analytically; trapping occurs in the first bucket of the wakefield ( $k_{p} \zeta \geq-2.5 \pi$ in Fig. 1) and the momentum of the electrons in the beatwave separatrices have a rather complex phasedependence, owing to the envelope of the drive laser pulse and the corresponding wakefield amplitude. Trapping requires the conditions $u_{b+} \geq u_{s}$ and $u_{b-} \leq u_{c f}$ to be satisfied, so that the beatwave can reach electrons in the cold fluid orbit and move them above the separatrix $\left(u_{b \pm}\right.$ is the maximum/minimum momentum on the beatwave separatrix defined previously, $u_{s}$ the momentum on the plasma wave separatrix, and $u_{c f}$ the momentum on the cold fluid orbit). The beat wave separatrix satisfying the conditions $u_{b+} \geq u_{s}$ and $u_{b-} \leq u_{c f}$ is shown in Fig. 2, for the case of $a_{0}=1.3$ (corresponding to the time-averaged, normalized intensity on axis $\left.\left\langle a_{0}^{2}\right\rangle(r=0)=0.85\right), \beta_{b}=-1 / 3$, and just at the threshold value of $a_{1}$ that allows trapping into the 1D separatrix (which occurs at one particular phase). The centroid position of the beatwave separatrix, represented as the $u_{b, a v}=\gamma_{b} \beta_{b} \gamma_{\perp}(0)$ curve in Fig. 2, is phase-dependent through its dependence on $a_{0}\left(k_{p} \zeta\right)$.

The trapping threshold was calculated for the particular case of $\beta_{b}=-1 / 3$ (which corresponds to a frequency-doubled counter-propagating beam, as will be the case for our examples in Sec. III); we calculated numerically, for each value of $a_{0}(r=0)$, the minimum value of $a_{1}$ that allows trapping of electrons from the cold fluid orbit into the $1 \mathrm{D}$ or $2 \mathrm{D}$ separatrix. The results for the case of a ring (1D separatrix) or Gaussian (2D separatrix) are shown in Fig. 3. The difference between the 1D and 2D cases increases as $\alpha a_{0}$ increases,

reaching more than two orders of magnitude for $\alpha a_{0}=\sqrt{2}$. This large difference is due to the fact that, while the 2D Hamiltonian does not depend on the wake amplitude, the 1D does; when $\phi_{0}$ tends to one, the 1D separatrix orbit approaches the cold fluid orbit, lowering the trapping threshold.

\section{Maximum momentum}

The maximum longitudinal momentum of a trapped and focused electron can also be calculated from the plasma wave Hamiltonian. We will first assume that the electron is 


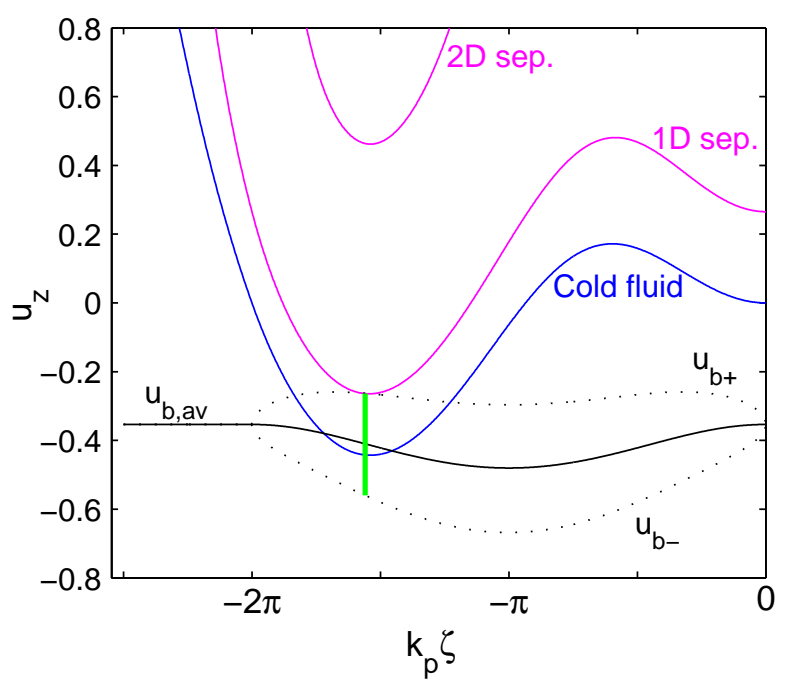

FIG. 2: Phase-space $\left(k_{p} \zeta, u_{z}\right)$ diagram for the trapping threshold calculation, with $\left\langle a_{0}^{2}\right\rangle(r=0)=$ 0.85 and plasma density $n_{e}=2 \times 10^{19} \mathrm{~cm}^{-3}$, for the case of trapping from the cold fluid orbit to the 1D separatrix. The difference between the maximum and minimum amplitudes of the beatwave separatrices $\left(u_{b+}\right.$ and $u_{b-}$, dashed curves) increases with increasing $a_{1}$, until trapping becomes possible (here, just at threshold, it would occur at one optimum phase, along the vertical line).

trapped after the first bucket (i.e., $k_{p} \zeta<-2.5 \pi$ ), as would be the case for the threepulse CPI scheme. For example, let us assume that trapping occurs in the second bucket $\left(-4.5 \pi<k_{p} \zeta<-2.5 \pi\right)$. Consider a trapped electron in a focusing phase, close to the defocused phase region (e.g., $k_{p} \zeta=-4 \pi$ and near $u_{z}=0$ on Fig. 1).

The electron may follow a trapped orbit near the separatrix until the point noted A on Fig. 1; it will then enter a defocusing phase. Note that the momentum at the point $\mathrm{A}$ is the same as the maximum momentum of the $2 \mathrm{D}$ separatrix, noted as point $\mathrm{B}$ on Fig. 1, $u_{A}=u_{B}=\beta_{g} \gamma_{g}^{2}\left(1 / \gamma_{g}+\alpha^{2} \phi_{0}\right)+\alpha \gamma_{g}^{2}\left[2 \phi_{0} / \gamma_{g}+\alpha^{2} \phi_{0}^{2}\right]^{1 / 2}$.

After the electron passes point A in Fig. $1\left(k_{p} \zeta=-4 \pi\right)$ and enters a defocusing phase region, it is not expelled out of the wakefield as one might expect. As the electron moves off-axis, it eventually moves to radii near the off-axis peak (at $r_{m}=r_{0} \sqrt{1-\alpha / \beta}$ ) such that $\partial a^{2} / \partial r<0$, and will be accelerated and focused at those radii. A trapped bunch will then form a ring at $r_{m}$. The maximum energy gain of the beam will be larger, since the peak accelerating field of the wakefield is larger off-axis.

This maximum value can be approximated as follows: i) the electron first propagates 


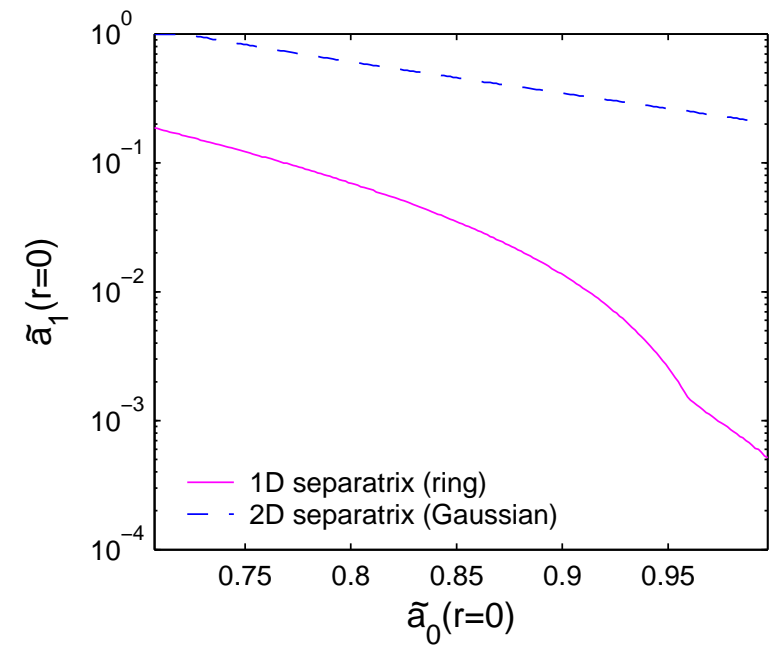

FIG. 3: Threshold value of the time-averaged colliding pulse vector potential $\tilde{a}_{1}=\sqrt{\left\langle a_{1}^{2}\right\rangle}=a_{1} / \sqrt{2}$ that allows trapping as a function of the time-averaged pump pulse vector potential $\tilde{a}_{0}(r=0)=$ $a_{0}(r=0) / \sqrt{2}$, for plasma density $n_{e}=2 \times 10^{19} \mathrm{~cm}^{-3}$ and $\beta_{b}=-1 / 3$, for both ring (solid curve) and Gaussian (dashed curve) transverse laser profiles.

on axis along the $1 \mathrm{D}$ separatrix until point $\mathrm{A}$, where it reaches the momentum value $u_{A}$; ii) then, we can assume that it will follow a 1D orbit off-axis; to simplify, we will assume that it propagates at $r=r_{m}$ (which leads to overestimate the maximum energy gain since an electron near axis would oscillate around $r_{m}$ ). The Hamiltonian on this off-axis orbit is simply $H_{R}=\left(1+u_{A}^{2}\right)^{1 / 2}-\beta_{g} u_{A}$. The wakefield potential in the ring, i.e., at $r=r_{m}$, is $\phi_{R}(\zeta)=\phi_{R 0} \sin \left(k_{p} \zeta\right)$, with $\phi_{R 0}=\phi_{0} \beta^{2} \exp [-2(1-\alpha / \beta)]$. The momentum of the electron on that orbit is then given by $u_{R}(\zeta)=\beta_{g} \gamma_{g}^{2}\left(H_{R}+\phi_{R}\right)+\gamma_{g}\left[\gamma_{g}^{2}\left(H_{R}+\phi_{R}\right)^{2}-1\right]^{1 / 2}$. The maximum momentum is reached at the phase where the potential is also maximum (i.e., $\phi_{R, \max }=\phi_{R 0}$ ), and is given by

$$
u_{R, \max }=\beta_{g} \gamma_{g}^{2}\left(H_{R}+\phi_{R, \max }\right)+\gamma_{g} \sqrt{\gamma_{g}^{2}\left(H_{R}+\phi_{R, \max }\right)^{2}-1}
$$

The same method can be applied to the case of an electron trapped in the first plasma wave bucket (for $k_{p} \zeta>-2.5 \pi$, as would be the case for the two-pulse CPI scheme). Let us consider an electron initially on axis, following the $1 \mathrm{D}$ separatrix until the phase $-2 \pi$; as before, we assume that the electron will then propagate off axis in the ring, on an orbit with the same Hamiltonian $H_{R}$ as previously defined. However the wakefield potential in the first bucket will be different, $\phi_{R}^{(1)}(\zeta)=\phi_{R 0}\left[1-\cos \left(k_{p} \zeta\right)-\left(k_{p} \zeta / 2\right) \sin \left(k_{p} \zeta\right)\right] / \pi$. Also, 
the laser electric field is present in the first bucket; consequently, we must account for the

transverse quiver of the electron: $u_{R}(\zeta)=\beta_{g} \gamma_{g}^{2}\left(H_{R}+\phi_{R}^{(1)}\right)+\gamma_{g}\left[\gamma_{g}^{2}\left(H_{R}+\phi_{R}^{(1)}\right)^{2}-\gamma_{\perp}^{2}\right]^{1 / 2}$, with $\gamma_{\perp}^{2}=1+a^{2} / 2$ (here we assume $a \simeq a(\zeta)$, which will be valid for $\gamma_{g} \gg 1$ ). The maximum momentum is reached when both the potential in the ring $\phi_{R}$ is maximum, and $\gamma_{\perp}$ is minimum (i.e., $\gamma_{\perp}=1$ for linear polarization). The maximum of the potential occurs at $k_{p} \zeta_{t} \simeq-4.49$, where it reaches the maximum value $\phi_{R, \max }=\phi_{R}\left(\zeta_{t}\right) \simeq 1.09 \phi_{R 0}$. The maximum momentum is then given by Eq. (11), with the maximum potential in the first plasma wake bucket, $\phi_{R, \max }$.

\section{NUMERICAL STUDIES}

In this section we present numerical examples of optical injection into wakefields excited by a higher-order mode drive laser. The next experiments in triggered injection will most likely use two-pulse CPI. The numerical examples presented in this section will therefore focus on this optical injection scheme. However, it should be emphasized that similar results can be obtained with the three-pulse CPI scheme, or with any other injection scheme, as the use of higher-order mode laser wakefields decreases the threshold for trapping regardless of the specific injection mechanism.

In particular, we consider two counterpropagating resonant laser pulses $\left(L_{1}=L_{2}=\right.$ $\left.\lambda_{p}\right)$, with half-sine longitudinal profiles and wavelengths $\lambda_{0}=0.8 \mu \mathrm{m}$ and $\lambda_{1}=0.4 \mu \mathrm{m}$. We consider different wavelengths to avoid a static beatwave, that will continuously push plasma electrons between fixed interference fringes, leading to the development of highlynonlinear density perturbations. We consider the simple case of a frequency-doubled counterpropagating laser pulse such that our fluid model remains valid.

The wakefield associated with the counterpropagating laser pulse is typically negligible since $a_{1}^{2} \ll a_{0}^{2}$. The ambient plasma density in the following examples is $n_{e}=2 \times 10^{19} \mathrm{~cm}^{-3}$. All numerical results are obtained by pushing test electrons (via an adaptive Runge-Kutta solver) in the specified fields of the lasers and plasma wakefield (cf. Sec. II A). 

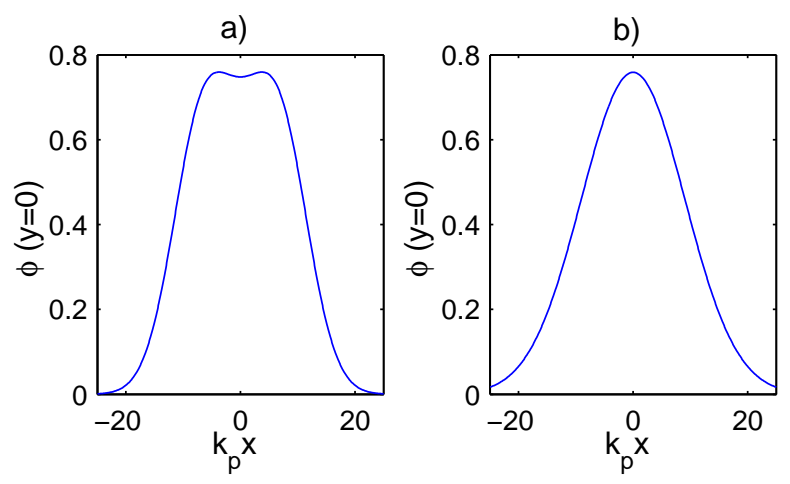

FIG. 4: Transverse profiles of the electrostatic plasma wakefield potential excited with the laser beam profiles: (a) ring beam with $\alpha=0.6$ and (b) Gaussian beam $(\alpha=1)$. Parameters have been chosen so that the laser power and peak wakefield amplitudes are the same for both cases.

\section{A. Comparison of a ring and a Gaussian laser profile}

In order to compare the relative performance using higher-order mode laser-driven wakefields with that of the usual Gaussian transverse laser beam profile, we consider two drive laser pulses with the same power $\left(P_{0}=30 \mathrm{TW}\right)$, and with transverse profiles that generate the same maximum peak amplitude of the wakefield. In the following example, we used $a_{0}=2.3$ and 1.39 and $r_{0}=13 \mu \mathrm{m}$ and $21.5 \mu \mathrm{m}$ for the ring and Gaussian beams, respectively. The ring profile is almost flat transversely, with $\alpha=0.6$. The time-averaged normalized intensities on axis for the ring and Gaussian beams are respectively, $\left\langle a_{0}^{2}\right\rangle(r=0)=\left(\alpha a_{0}\right)^{2} / 2$ $=0.95$ and 0.97 .

The maximum peak amplitude of the wakefield is then the same for both cases, $\phi_{0}=$ 0.76 (on-axis for the Gaussian and off-axis for the ring). The two corresponding wakefield potential profiles are shown in Fig. 4. The counter-propagating injection laser beam is the same in both cases, Gaussian transverse profile with $a_{1}=0.01$, spot size of $r_{1}=15 \mu \mathrm{m}$ $\left(P_{1}=3 \mathrm{GW}\right)$, and $0.4 \mu \mathrm{m}$ wavelength (frequency-doubled of the drive beam).

Test electrons are loaded in front of the drive pulse, filling a cylindrical volume of length $\lambda_{p}$ and radius $\sigma_{r 0}$, chosen such that no trapping can occur for electrons loaded at a radius $r>\sigma_{r 0}$. The initial positions of the pulses and test particles are chosen such that the two colliding pulses overlap the test particles (at the time of overlap, the pulses and particles are all within a region of length $\lambda_{p}$ in phase space).

The numerical parameters and main numerical results are reported in Table I. The 
transverse wakefield structure has a strong effect on the amount of trapped charge; almost 70 times more charge is trapped in the ring wakefield than in the Gaussian wakefield.

It should be noted that according to the results from the previous section (Fig. 3), there should be no trapping at all for the Gaussian case for this value of $a_{1}$. But, since the beam radius has been chosen relatively large (in order to avoid diffraction effects), the trapped electrons experience a broad wake with weak focusing forces. The electrons exiting the beatwave start to follow orbits between the 1D and 2D separatrices; when the trapped electrons enter the defocusing region $\left(-2 \pi>k_{p} \zeta>-2.5 \pi\right.$ on Fig. 1), those with the lowest longitudinal momentum will drift off-axis and be lost, but those at the highest longitudinal momentum (close to the 2D separatrix) will not drift enough to be lost (since the transverse forces remain weak near $-2 \pi)$. Those electrons surviving to $k_{p} \zeta \geq-2 \pi$ will be focused again and can form a trapped bunch.

In addition to the difference in trapped charge, the beam quality is improved by injection into the ring structure, as shown in Fig. 5. The results show that for the Gaussian wakefield, electrons are continuously injected into the trapped orbits, and the resulting beam can have a relatively large energy spread and emittance. In addition to increased maximum beam energy, which is higher for the ring beam $\left(\langle\gamma\rangle_{\max } \simeq 286\right.$, compared to $\langle\gamma\rangle_{\max } \simeq 230$ for the Gaussian beam), the relative beam energy spread reaches a minimum of about $5.5 \%$ (compared to almost 30\% near maximum energy for the Gaussian wakefield), and the emittance grows to approximately $2 \mu \mathrm{m}$ rad (compared to $30 \mu \mathrm{m}$ rad for the Gaussian beam). The maximum value of the beam momentum using a ring profile is in good agreement with the estimates from the Hamiltonian analysis [Eq. (11)], which gives $u_{R, \max } \simeq 292$ for these parameters.

\section{B. Creation of a ring beam}

Higher-order modes could also be used to shape the transverse profile of the electron beam. In particular, it is possible to create transversely ring-shaped beams if the $\alpha$ parameter in the higher-order laser mode [cf. Eq. (1)] is small enough to prevent any particle from remaining near the axis when the beam goes beyond the phase $k_{p} \zeta \geq-2 \pi$ (cf. Fig. 1). A potential application of such ring beams could be for an ion channel laser (ICL) [20, 21]. A complication of the ICL is that, for an electron beam injected on-axis into an 
TABLE I: Summary of numerical parameters and results. The plasma density was $n_{e}=2 \times$ $10^{19} \mathrm{~cm}^{-3}\left(\lambda_{p}=7.5 \mu \mathrm{m}\right)$.

\begin{tabular}{ccc}
\hline \hline & Ring profile & Gaussian profile \\
\hline$\alpha$ & 0.6 & 1 \\
$\left\langle a_{0}^{2}\right\rangle(r=0)$ & 0.95 & 0.97 \\
$P_{0}(\mathrm{TW})$ & 30 & 30 \\
$P_{1}(\mathrm{GW})$ & 3 & 3 \\
$\phi_{\max }$ & 0.76 & 0.76 \\
$\lambda_{0}(\mu \mathrm{m})$ & 0.8 & 0.8 \\
$\lambda_{1}(\mu \mathrm{m})$ & 0.4 & 0.4 \\
$Q_{\text {trap }}(\mathrm{pC})$ & 26 & 0.4 \\
\hline \hline
\end{tabular}

ion channel, the effective wiggler parameter $a_{\beta}$ is proportional to the particle's transverse oscillation amplitude [21]; hence, it varies from zero for the electrons on-axis to a maximum value for the electrons farthest off-axis (typically, $a_{\beta} \gg 1$ ). This effect results in a broad spectrum of emitted radiation and inhibits the self-amplified spontaneous emission process. A solution to this problem is to use a ring beam; in that case, the wiggler parameter would be approximately equal for all the electrons in the beam, and, hence, the synchrotron radiation would be peaked at one wavelength depending on the energy of the electrons and the radius of the ring.

Generation of such a ring beam can be accomplished by using a higher-order mode laserdriven wakefield. Consider a transverse beam profile with $\alpha=0.3$. The laser power is $P_{0}=38.6 \mathrm{TW}$, with a radius $r_{0}=13.5 \mu \mathrm{m}\left(a_{0}=2.5\right.$, or $\left.\left\langle a_{0}^{2}\right\rangle(r=0)=0.28\right)$, giving a peak wakefield amplitude $\phi_{\max }=0.68$ (off-axis). The resulting wakefield amplitude is shown in Fig. 6. As in the previous section, we consider generation of the electron beam via the two-pulse CPI method; the counterpropagating laser beam has a Gaussian transverse profile with $a_{1}=0.07$ and $r_{1}=20 \mu \mathrm{m}\left(P_{1}=0.27 \mathrm{TW}\right)$.

The evolution of the beam properties of the electron beam generated with these laser parameters is shown in Fig. 7. The beam quality is not as good as that produced by the near-flat transverse laser beam profile (cf. Fig. 5): the normalized emittance is about 20$30 \mu \mathrm{m} \mathrm{rad}$ and the minimum relative energy spread is about $20 \%$. Injecting this beam into a 

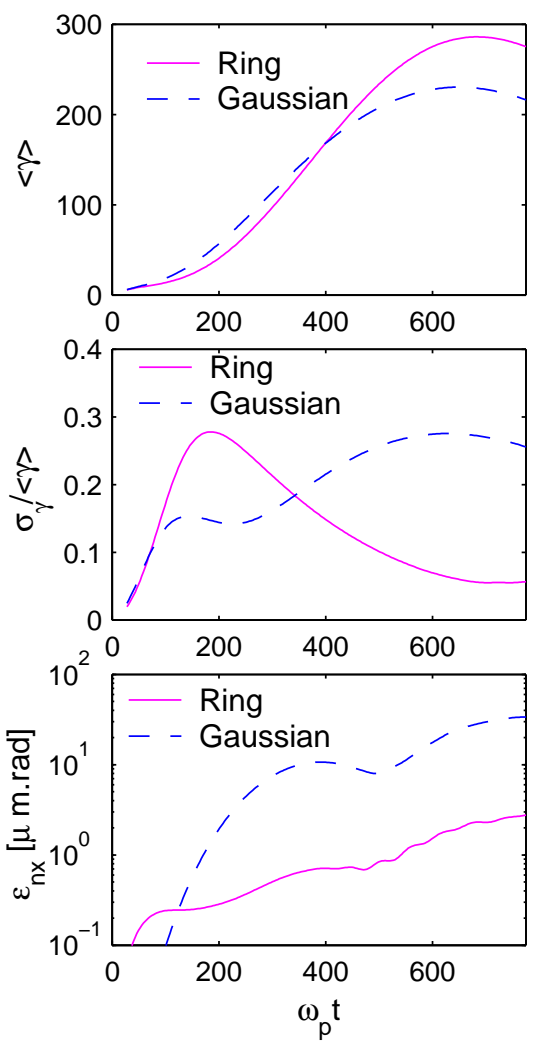

FIG. 5: Mean energy $\langle\gamma\rangle$, relative energy spread $\sigma_{\gamma} /\langle\gamma\rangle$, and normalized transverse emittance $\epsilon_{n x}$ ( $\mu \mathrm{m} \mathrm{rad)} \mathrm{for} \mathrm{the} \mathrm{ring} \mathrm{(solid} \mathrm{curves)} \mathrm{and} \mathrm{Gaussian} \mathrm{(dashed} \mathrm{curves)} \mathrm{beams} \mathrm{defined} \mathrm{in} \mathrm{Table} \mathrm{I.}$

second accelerator stage could increase the maximum energy and decrease the relative energy spread. The maximum value of the beam momentum for this example is $\left\langle u_{z}\right\rangle_{\max } \simeq 161$; and the estimation from the Hamiltonian analysis [Eq. (11)] gives $u_{R, \max } \simeq 184$. The amount of charge in the beam for this example is relatively high, about $42 \mathrm{pC}$. Beam loading effects should however remain negligible for these parameters [11].

The evolution of the electron beam transverse density profile is shown in Fig. 8. At $\omega_{p} t=300$, i.e., near $k_{p} \zeta=-2 \pi$ on Fig. 1 , the electron beam remains mostly focused on axis but starts to defocus and expand radially. Then, the beam evolves into a ring structure near the point of maximum energy and minimum relative energy spread of the beam, $\omega_{p} t \simeq 630$.

Figure 9 shows the results of a numerical calculation using a tightly focused counterpropagating beam. The drive beam is the same as in the previous case $\left(P_{0}=38.6 \mathrm{TW}\right)$, but the colliding beam is $0.21 \mathrm{TW}$ with $r_{1}=5 \mu \mathrm{m}$ and $a_{1}=0.25$. In the previous case most of the initial trapping occurred off-axis (where $a_{0} a_{1}$ is maximum); the tight focusing of the injection beam provides injection near axis. Figure 9 shows the $(\gamma, r)$ phase space at $\omega_{p} t=30$; the 


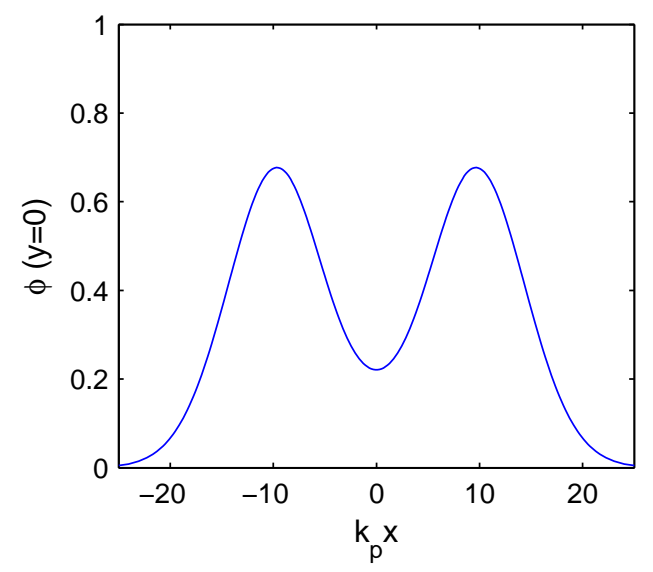

FIG. 6: Transverse profile of a wakefield generated by a ring laser beam with $\alpha=0.3,\left\langle a_{0}^{2}\right\rangle(r=0)$ $=0.28$, and $r_{0}=13.5 \mu \mathrm{m}\left(P_{0}=38.6 \mathrm{TW}\right)$.

energy exhibits a quadratic dependence on transverse position. The correlation arises from the transverse wakefield structure, where the accelerating gradient is stronger off-axis. An electron beam with this energy-radius correlation is naturally conditioned for application in a free-electron laser $[22,23]$. The degree of conditioning could be adjusted by varying the transverse profile of the laser exciting the wake.

\section{DISCUSSION}

The use of high-intensity laser pulses with higher-order transverse modes as the driver in a wakefield accelerator has been consider as a method to dramatically improve the efficiency of electron trapping in the plasma wave. Linear theory predicts that the wake potential is proportional to the transverse profile of the normalized intensity profile $\left(\sim a^{2}\right)$. Hence, the radial focusing field of the wake is proportional to $\partial a^{2} / \partial r$. Typically, for a Gaussian drive laser profile, the trailing section of the wake "phase bucket" (consisting of trapped orbits lying close to the 1D separatrix) is accelerating but defocusing. Hence, to trap electrons with a Gaussian drive beam requires injecting electrons at higher energies such that they are trapped more deeply on "inner" orbits within the phase bucket. On the contrary, by using higher-order transverse modes to create a ring drive laser with a maximum intensity off-axis $\left(\partial a^{2} / \partial r>0\right.$ near axis), the phase regions of the wake that are focusing and defocusing can be completely reversed in comparison to a Gaussian drive laser. This implies that the trapped 

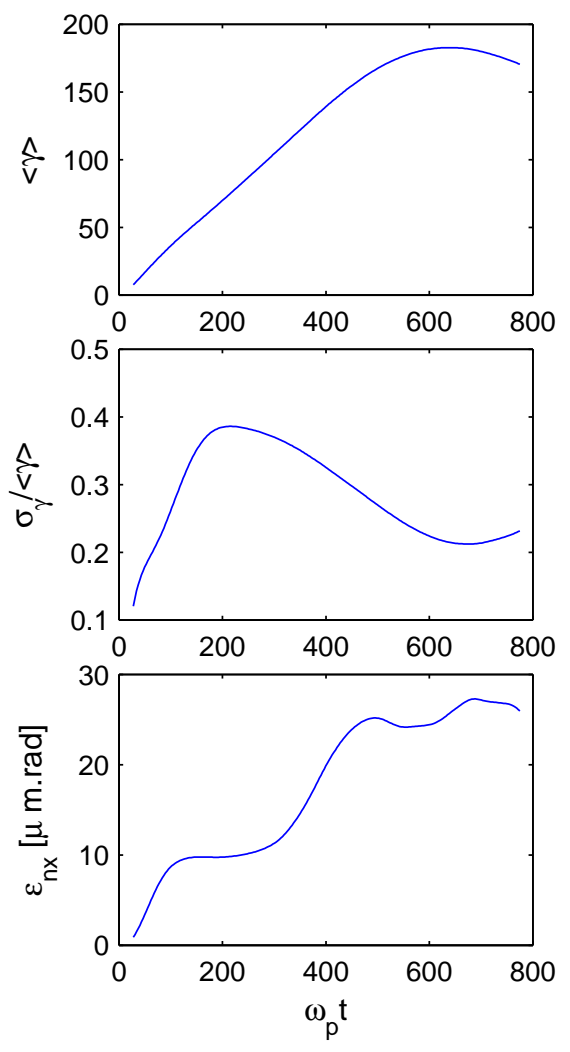

FIG. 7: Beam properties (mean energy $\langle\gamma\rangle$, relative energy spread $\sigma_{\gamma} /\langle\gamma\rangle$, and normalized transverse emittance $\epsilon_{n x}$ ) of a ring electron beam generated by a higher-order mode laser beam with $\alpha=0.3,\left\langle a_{0}^{2}\right\rangle(r=0)=0.28$, and $r_{0}=13.5 \mu \mathrm{m}\left(P_{0}=38.6 \mathrm{TW}\right)$.

orbits in the trailing region of the phase bucket, lying closest to the $1 \mathrm{D}$ separatrix, are now accelerating and focusing. Consequently, electrons can be injected onto these trapped and focused orbits with a minimum energy, e.g., a substantially lower energy in comparison to a Gaussian driver. The effect of this is to significantly reduce the threshold for electron trapping in the wakefield, resulting in an enhanced yield of energetic electrons for a wide variety of self-trapping and laser-triggered injection mechanisms.

The analysis presented in this paper assumed a wakefield response as predicted by linear theory, which is only rigorously valid in the regime $a^{2} / 2 \ll 1\left(\phi^{2} \ll 1\right)$. Nevertheless, the linear expression for the wake is expected to be an adequate approximation to illustrate the benefits of using higher-order laser modes even in the mildly nonlinear limit in which $a^{2} / 2$ approaches unity. A related constraint on the laser intensity is that the plasma is assumed to not be in the blowout regime. To estimate the laser intensity at which the onset of blowout 

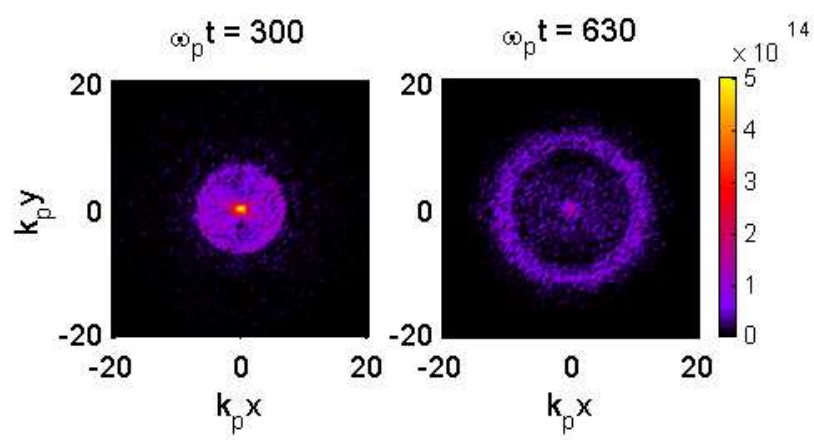

FIG. 8: Transverse profile of the electron beam represented as surface densities (in units of $\mathrm{cm}^{-2}$ ), at $\omega_{p} t=300$ and $\omega_{p} t=630$.

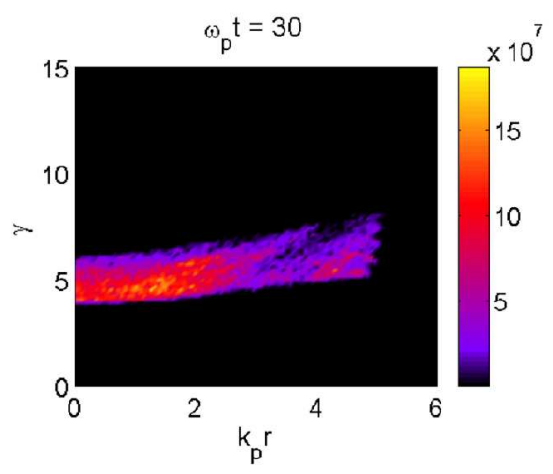

FIG. 9: $(\gamma, r)$ phase space at $\omega_{p} t=30$, represented as a density (in units of $\mu \mathrm{m}^{-1} \mathrm{MeV}^{-1}$ ), for a tightly focused counterpropagating beam with $r_{1}=5 \mu \mathrm{m}$ and $a_{1}=0.25$.

occurs, consider a long (compared to the plasma wavelength) pulse. In the long pulse regime, the plasma density response [1] is given approximately by $n / n_{0} \simeq 1+k_{p}^{-2} \nabla_{\perp}^{2}\left(1+a^{2} / 2\right)^{1 / 2}$. For a Gaussian drive pulse of the form $a^{2}=a_{0}^{2} \exp \left(-2 r^{2} / r_{0}^{2}\right)$, the density response along the axis $(r=0)$ in the limit $a^{2} / 2 \ll 1$ is given by $n_{0} / n \simeq 1-a_{0}^{2} /\left(2 k_{p}^{2} r_{0}^{2}\right)$. Hence, avoiding blowout requires $a_{0}^{2} / 2<k_{p}^{2} r_{0}^{2}$, i.e., moderate intensity and sufficiently large spot size (this condition is satisfied for the examples given above). A rigorous study of the effect of higherorder laser drivers on electron trapping in the nonlinear regime requires the use a numerical model of wake generation, such as a nonlinear fluid code.

In this paper, the benefits of using higher-order laser drivers were illustrated by application to the specific case of laser triggered injection using the two-pulse colliding pulse injection method. Although this paper only considers application to the two-pulse colliding pulse injection method in detail, the use of higher-order laser drivers is expected to enhance 
trapping and electron beam generation in a wide variety of self-trapping and laser injection mechanisms. Numerical studies of two-pulse colliding pulse injection, presented in Sec. III, show a significant (approximately a factor of 70) increase in the trapped charge and improved bunch quality (cf. Fig. 5) by using wakefields excited by a higher-order mode laser compared to using wakefields driven by a laser pulse with a Gaussian transverse profile. The relative beam energy spread and normalized transverse emittance remain relatively good, although an increase in the transverse normalized emittance is observed when the trapped electrons reach the phase region where focusing occurs off-axis (at the maximum wakefield amplitude). A high-quality trapped electron beam could be extracted before reaching this phase region (at $\omega_{p} t \approx 300$ in the example of Sec. III). This mechanism can also produce a radius-energy correlation that could be of interest for beam conditioning applications to free electron lasers [23]. The wakefield in the phase region of off-axis focusing $\left(-4 \pi<k_{p} \zeta<-3 \pi\right.$ in Fig. 1) gathers the electrons off-axis at a radius where the wakefield amplitude is maximum. The acceleration can continue (in the off-axis focused region), leading to higher energy

electron beams. This generates novel ring-shaped electron beams (cf. Fig. 8), that could be envisioned for applications such as the ion-channel laser [22].

\section{Acknowledgments}

Pierre Michel is the recipient of the Andrew Sessler Postdoctoral Fellowship at LBNL. This work was supported by the Director, Office of Science, Office of High Energy Physics, of the U.S. Department of Energy under Contract No. DE-AC02-05CH11231.

[1] E. Esarey, P. Sprangle, J. Krall, and A. Ting, IEEE Trans. Plasma Sci. 24, 252 (1996).

[2] C. G. R. Geddes, C. Toth, J. van Tilborg, E. Esarey, C. B. Schroeder, D. Bruhwiler, C. Nieter, J. Cary, and W. P. Leemans, Nature 431, 538 (2004).

[3] J. Faure, Y. Glinec, A. Pukhov, S. Kiselev, S. Gordienko, E. Lefebvre, J. P. Rousseau, F. Burgy, and V. Malka, Nature 431, 541 (2004).

[4] S. P. D. Mangles, C. D. Murphy, Z. Najmudim, A. G. R. Thomas, J. L. Collier, A. E. Dangor, E. J. Divall, P. S. Foster, J. G. Gallacher, C. J. Hooker, et al., Nature 431, 535 (2004).

[5] D. Umstadter, J. K. Kim, and E. Dodd, Phys. Rev. Lett. 76, 2073 (1996). 
[6] E. Esarey, R. F. Hubbard, W. P. Leemans, A. Ting, and P. Sprangle, Phys. Rev. Lett. 79, 2682 (1997).

[7] S. Bulanov, N. Naumova, F. Pegoraro, and J. Sakai, Phys. Rev. E 58, 5257 (1998).

[8] C. B. Schroeder, P. B. Lee, J. S. Wurtele, E. Esarey, and W. P. Leemans, Phys. Rev. E 59, 6037 (1999).

[9] E. Esarey, C. B. Schroeder, W. P. Leemans, and B. Hafizi, Phys. Plasmas 6, 2262 (1999).

[10] H. Suk, N. Barov, J. B. Rosenzweig, and E. Esarey, Phys. Rev. Lett. 86, 1011 (2001).

[11] G. Fubiani, E. Esarey, C. B. Schroeder, and W. P. Leemans, Phys. Rev. E 70, 016402 (2004).

[12] H. Kotaki, S. Masuda, M. Kando, J. K. Koga, and K. Nakajima, Phys. Plasmas 11, 3296 (2004).

[13] G. Fubiani, E. Esarey, C. B. Schroeder, and W. P. Leemans, Phys. Rev. E 73, 026402 (2006).

[14] P. Sprangle, E. Esarey, A. Ting, and G. Joyce, Appl. Phys. Lett. 53, 2146 (1988).

[15] B. A. Shadwick, G. M. Tarkenton, and E. H. Esarey, in Advanced Accelerator Concepts, edited by V. Yakimenko (AIP, New York, 2004), pp. 449-455.

[16] C. B. Schroeder, E. Esarey, and B. A. Shadwick, Phys. Rev. E 72, 055401 (2005).

[17] E. Esarey and W. P. Leemans, Phys. Rev. E 59, 1082 (1999).

[18] A. Ting, E. Esarey, and P. Sprangle, Phys. Fluids B 2, 1390 (1990).

[19] E. Esarey and M. Pilloff, Phys. Plasmas 2, 1432 (1995).

[20] D. H. Whittum, A. M. Sessler, and J. M. Dawson, Phys. Rev. Lett. 64, 2511 (1990).

[21] E. Esarey, B. A. Shadwick, P. Catravas, and W. P. Leemans, Phys. Rev. E 65, 056505 (2002).

[22] A. M. Sessler, D. H. Whittum, and L.-H. Yu, Phys. Rev. Lett. 68, 309 (1992).

[23] C. B. Schroeder, E. Esarey, and W. P. Leemans, Phys. Rev. Lett. 93, 194801 (2004). 